\title{
Kompetenzfelder der Elektronik - Systemlösungen des Instituts Electronic Engineering der FH JOANNEUM
}

H. Berger, C. Netzberger IEEE, W. Stocksreiter, R. Estrada-Vázquez, N. Blasonig, A. Preda, W. Obermayr, M. Salloker, C. Vogel IEEE

\begin{abstract}
Die verschiedenen Gebiete der Elektronik sind vielschichtig und umfangreich, sodass es einer zielgerichteten Strategie bedarf, um systemische Lösungen in verschiedenen Kompetenzbereichen anbieten zu können. Das Institut für Electronic Engineering an der FH JOANNEUM kann auf 25 Jahre F\&E-Aktivitäten zurückblicken, wobei es folgende Kompetenzbereiche systematisch bündelt: Im Bereich EMV fokussiert sich die Forschung auf die Prüfung elektromagnetischer Verträglichkeit und die Entwicklung von Funkapplikationen im Bereich Leistungselektronik, Kommunikation, Medizintechnik und Automatisierung. Das Forschungsgebiet ist stark mit dem Schwerpunkt Leistungselektronik verknüpft, bei dem zusätzlich noch intensiv an den Themen Simulation auf Schaltungs- und Systemebene, elektronischer Schaltungstechnik und Mechatronik mit Prototypenfertigung geforscht wird. Möglich wird das durch nachhaltige und strategische Investitionen in die dafür notwendige Laborinfrastruktur sowie durch einen mit der Industrie abgestimmten Kompetenzaufbau. Der Artikel geht auch auf unsere Kompetenzbereiche PCB-Design, FPGA-Design und Realtime Computing ein, die unsere übrigen Forschungsaktivitäten ergänzen und sowohl für zahlreiche Kundenprojekte als auch für den Wissenstransfer in die Lehre unabdingbar sind.
\end{abstract}

Schlüsselwörter: Leistungselektronik; Realtime Computing; FPGA; elektromagnetische Verträglichkeit

Fields of competence in electronics - system solutions at the Institute of Electronic Engineering FH JOANNEUM.

The various fields in electronics are complex and wide. Thus a goal-oriented strategy is necessary in order to provide systematical solutions in different fields of competence. For 25 years, the Institute of Electronic Engineering at FH JOANNEUM has been dealing with R\&D activities, thereby focusing systematically on the following fields of competence: research on electromagnetic compatibility (EMC) with applications in power electronics, communication, medical engineering and automation. The focus of power electronics lies on design, simulation and development of efficient power electronic components as well as on their applications in mechatronics and resource-saving systems. This is enabled via an effective and strategic investigation into the infrastructure equipment necessary and an establishment of competence in coordination with industry. In this article we discuss our fields of competence in power electronics, FPGA-design, realtime computing and EMC. They supplement our activities in R\&D and are crucial for customer projects as well as for the transfer of knowledge into education.

Keywords: power electronics; realtime computing; FPGA; electromagnetic compatibility

Eingegangen am 11. September 2019, angenommen am 21. Oktober 2019, online publiziert am 17. Dezember 2019 (c) The Author(s) 2019

\section{Einleitung}

Seit der Gründung des Studienganges Industrielle Elektronik im Jahre 1995 fand eine lebhafte Entwicklung in Hinblick auf Hochschulausbildung und F\&E am Institut für Electronic Engineering [1] der FH JOANNEUM statt: Bachelor- und Masterstudiengänge, ein Transferzentrum (Forschungsabteilung), das bereits auf eine erfolgreiche langjährige F\&E Aktivität verweisen kann und ein großes Netzwerk mit namhaften Unternehmen und Institutionen im In- und Ausland, wie AVL List GmbH oder Univerza v Ljublijani sind entstanden. Thematisch haben sich dabei zahlreiche Fachbereiche ergeben, wie z.B. Elektronische Systeme, Leistungselektronik, Realtime Computing, Programmierbare Logik (FPGA), Elektromagnetische Verträglichkeit, Automotive Control und Automatisierung. In diesem Artikel wird auf einige Fachbereiche näher eingegangen, auf F\&E-Aktivitäten, auf die Vernetzung mit der einschlägigen Elektronik-Industrie, auf Kooperationsprojekte mit Partnerunternehmen wie Infineon Technologies AG oder AT\&S AG und schließlich, damit verbunden, den Wissenstransfer in die Ausbildung.

\section{JOANNEUM Power Electronics Center (JPEC)}

Die fortschreitende Elektrifizierung aller Bereiche der Energieversorgung und Energieanwendung erfordert eine Vielzahl an Umformungen der elektrischen Energie. Die neuen LeistungsHalbleitertechnologien (SiC-, GaN-basierend) ermöglichen dabei eine wesentliche Steigerung des Umwandlungswirkungsgrades. Ein-

Berger, Hubert, FH JOANNEUM Electronic Engineering, Werk-VI-Straße 46, 8605 Kapfenberg, Österreich; Netzberger, Christian, FH JOANNEUM Electronic Engineering, Werk-VI-Straße 46, 8605 Kapfenberg, Österreich; Stocksreiter, Wolfgang, FH JOANNEUM Electronic Engineering, Werk-VI-Straße 46, 8605 Kapfenberg, Österreich; Estrada-Vázquez, Raúl, FH JOANNEUM Electronic Engineering, Werk-VI-Straße 46, 8605 Kapfenberg, Österreich; Blasonig, Nina, FH JOANNEUM Electronic Engineering, Werk-VI-Straße 46, 8605 Kapfenberg, Österreich; Preda, Aida, FH JOANNEUM Electronic Engineering, Werk-VI-Straße 46, 8605 Kapfenberg, Österreich; Obermayr, Werner, FH JOANNEUM Electronic Engineering, Alte Poststraße 147, 8020 Graz, Österreich (E-Mail: werner.obermayr@fh-joanneum.at); Salloker, Michael, FH JOANNEUM Electronic Engineering, Alte Poststraße 147, 8020 Graz, Österreich; Vogel, Christian, FH JOANNEUM Electronic Engineering, Alte Poststraße 147, 8020 Graz, Österreich 
sparungen bis zu $10 \%$ des Gesamtstromverbrauches sind dabei realistisch. Der Einsatz der neuen Halbleiter stellt allerdings die Entwicklungsingenieure vor große Herausforderungen. Das extrem schnelle Schalten mit sehr hohen Flanken (schnelle Spannungs-und Stromänderungen) führt in Verbindung mit parasitären Kapazitäten und Induktivitäten zu vielen oft nur schwer beherrschbaren Effekten. Diese neuen Herausforderungen können nur mithilfe der Anwendung von Methoden der Hochfrequenztechnik, im Besonderen der zugehörigen Messtechnik, bewältigt werden

An der FH JOANNEUM begann der Kompetenzaufbau in der Leistungselektronik vor etwa zehn Jahren. Damals wurde erkannt, dass die Leistungselektronik aufgrund neuer Wide-Bandgap Transistoren ( $\mathrm{SiC}$ und $\mathrm{GaN}$ ) vor großen Veränderungen steht. Mit unserem damals bereits etablierten EMV-Labor und den Aktivitäten in der Hochfrequenztechnik bestanden zudem beste Voraussetzungen, um den Leistungselektronik-Schwerpunkt mit Fokus auf miniaturisierte, hoch-taktende Konverter einzurichten. Es zeichnete sich auch bereits ab, dass die zukünftige Leistungselektronik enorme Packungsdichten erreichen wird und sich damit das Thema Electronic Packaging mit neuen Ansätzen als wesentliches Erfolgskriterium herauskristallisieren wird. Klassische Aufbautechniken im Schaltschrank werden dann nur mehr bei ganz hohen Leistungen zum Einsatz kommen.

2014 startete zu diesem Forschungsfeld das von der Programmlinie Research Studio Austria der FFG geförderte Projekt „JPEC " [2], in dem ein umfassender Kompetenzausbau auf Gebieten wie Schaltungs- und Systemsimulation, Advanced Packaging aber auch in ergänzenden Gebieten wie FPGA-basierter EchtzeitRechenplattformen stattfand.

Das Vorhaben zielte darauf ab, mit optimierten Schaltungstopologien und Advanced packaging Methoden generische Systemkomponenten bereitzustellen, die den Umstieg der Unternehmenspartner auf hocheffiziente Leistungsumformer in sehr breiten Anwendungsgebieten (von der Stromerzeugung und -verteilung, über Antriebstechnik für Transport und Industrie bis hin zu metallurgischen Prozessen) unterstützen.

Das JOANNEUM Power Electronics Center ist mittlerweile eng mit der einschlägigen Elektronik-Industrie vernetzt. In den Kooperationsprojekten werden zahlreiche SiC- und GaN-basierte Leistungskonverter für unterschiedliche Applikationsfelder entwickelt. Es entstehen typischerweise funktionelle Labormuster, an Hand derer sämtliche grundlegenden Fragestellungen geklärt werden. Diese Erkenntnisse fließen dann in die eigentlichen Produktentwicklungen der Partnerunternehmen ein. Das Team des JOANNEUM Power Elec tronic Centers begleitet diese Produktentwicklungen in Form von z.B. weiteren Simulationen oder der Durchführung von speziellen Mess- und Prüfaufgaben

\section{Einige Projektbeispiele}

\section{- 120 kW SiC-Inverter für Hochdrehzahlmotoren}

In Automobilanwendungen (Kooperatives Forschungsprojekt INTEGRA im Rahmen des Förderprogramms Mobilität der Zukunft)

- 3 kW GaN-basierter DC-DC Konverter

zur galvanisch getrennten Kopplung von $48 \mathrm{~V}$ mit $12 \mathrm{~V}$ Boardnetzen. Dabei handelt es sich um einen Resonanzkonverter mit ultrakompaktem $1 \mathrm{MHz}$ Planar-Transformer.

- 20 kW GaN-basierter DC-DC-Konverter zur Kopplung eines Brennstoffzellenstacks mit der Fahrzeugbatterie.

- 440 kVA SiC-basierter hochkompakter Netzwechselrichter Hierbei handelt es sich um einen Netzwechselrichter mit Interleaving-Technologie und gekoppelten Induktivitäten mit sehr hoher Leistungsdichte.
Das Leistungselektroniklabor wird aktuell zu einem Entwicklungsund Testlabor für Komponenten der Elektromobilität ausgebaut. Das Labor soll die bereits sehr intensiven Forschungs- und Entwicklungstätigkeiten im Bereich elektrifizierter Antriebsstränge samt (Lade-)Infrastruktur umfassend unterstützen. Die Testmethodik des Labors wird konsequent auf Hardware-in-the-Loop (HiL) Konzepten beruhen, bei denen die reale Umgebung der zu testenden Komponenten vollständig (leistungs-) elektronisch nachgebildet wird. Unterschiedliche Funktionalitäten werden alleine durch Einspielen der entsprechenden Software-Modelle ausgewählt.

Die am Institut entwickelten Multiprozessor- bzw. FPGA-Hochleistungs-Rechenplattformen sind dabei in der Lage, die Modelle in Echtzeit zu verarbeiten. Zykluszeiten im Bereich von $1 \mu \mathrm{s}$ ermöglichen es, die physikalischen Eigenschaften z.B. von Batterien ohne Genauigkeitsverlust nachzubilden.

Das Testcenter wird darüber hinaus mit dem bestehenden Labor für elektromagnetische Verträglichkeit verbunden, sodass die funktionellen Tests mit der Überprüfung der elektromagnetischen Verträglichkeit in allen Arbeitspunkten des realen Betriebs kombiniert werden können. Mittels zirkulierenden Energieflusses sollen Leistungen bis mindestens $440 \mathrm{~kW}$ ermöglicht werden.

\section{Modellbasiertes Realtime Computing}

Bei der Anwendung von Realtime Computing in der Leistungselektronik haben wir in den letzten Jahren die Notwendigkeit erkannt, die Flexibilität der modellbasierten Softwareentwicklung (Model Based Design, MBD) mit den neuesten ultraschnellen Echtzeitumgebungen zu kombinieren. Dieser Bedarf erfordert hohe dynamische Kontroll-Anforderungen im Zusammenhang mit den miniaturisierten, hochmodernen Leistungswandlern in einem optimierten Entwicklungsprozess.

Verschiedene Aktivitäten im Bereich der Soft- und Hardwareentwicklung, wie die von der Planung über die Implementierung bis hin zum Test, sind durch MBD in einer einzigen Programmierumgebung gebunden. Diese Integration beschleunigt naturgemäß den Entwicklungsprozess und spart Ressourcen, wodurch die Popularität dieser Strategie in den vergangenen Jahren stark gestiegen ist [3]. Zusätzlich ist eine Echtzeitumgebung notwendig, die den Erfordernissen der hohen dynamischen Leistungswandler entspricht. Solche Voraussetzung sind:

- Niedrige Latenzzeit: Das „Alter" von Sensoren gesammelter Daten sollte so niedrig wie möglich sein.

- Schnelle Exekutionszeiten: Die Geschwindigkeit eines Algorithmus sollte um ein Vielfaches schneller sein als die Zeitkonstante des kontrollierten Systems oder einer Emulation (z.B. eines schnellen Motors)

- Genauigkeit des Berechnungszyklus: Sicherstellung, dass ein implementierter Algorithmus während eines Berechnungszyklus vollständig ausgeführt wird.

- Schneller Datentransfer: ist für den Austausch von Daten zwischen verschiedenen Geräten notwendig (distributed realtime computing)

- Synchrone Datenverwaltung: Daten des gleichen Zeitstempels sollen von der Recheneinheit synchron erhalten und gesendet werden.

All diese Anforderungen werden von Field Programmable Gate Arrays (FPGA) erfüllt. Diese Vorteile werden allerdings im Vergleich mit Mikroprozessoren von längeren Entwicklungszeiten der Steueralgorithmen begleitet. Um einen Trade-off zwischen diesen beiden Welten zu finden, haben heute sogenannte All-Programmable System-on-Chip (SoC) den Markt erobert. Die erfolgreichste Familie 


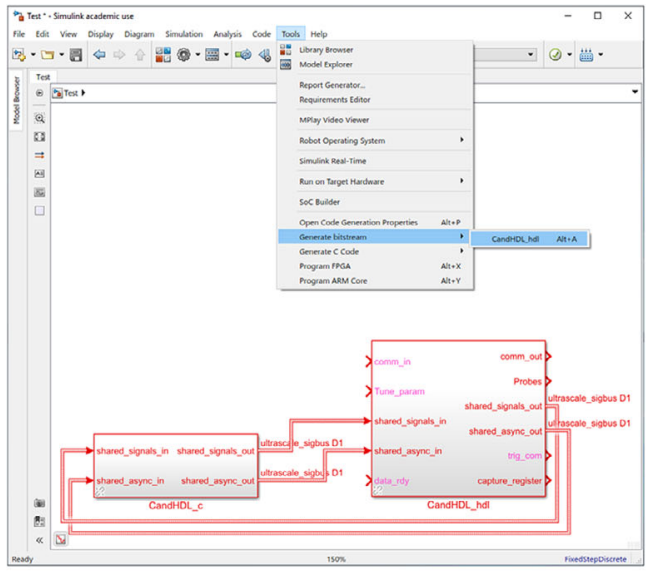

(a)

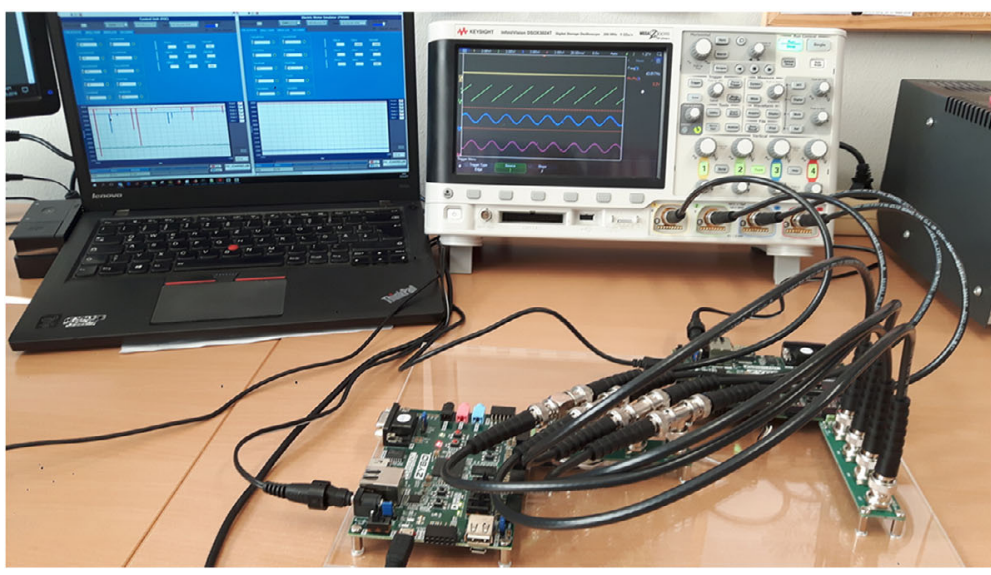

(b)

HIL DEMONSTRATOR

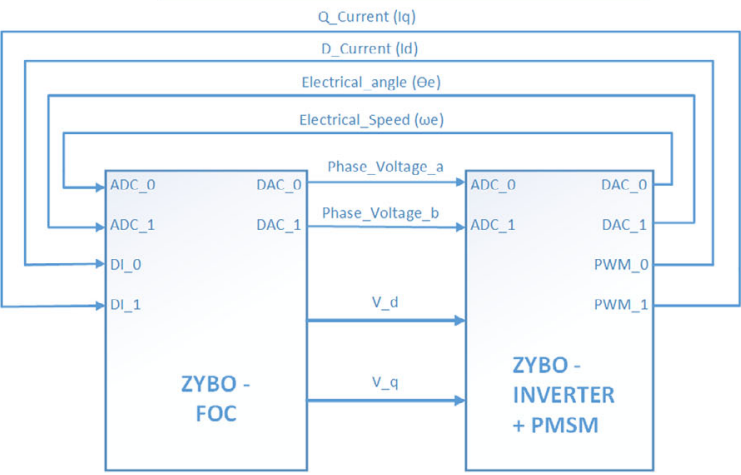

(c)

Abb. 1. Hardware-in-the-Loop (HIL) Demonstrator einer Permanentmagnet-Synchronmaschine (PMSM) und des Controllers basierend auf Field Oriented Control (FOC). (a) Implementierung und Verwaltung von Modellen in MATLAB/Simulink ${ }^{\circledR}$, (b) Testaufbau und (c) Blockschaltbild der Verbindungen

ist ZYNQ von Xilinx; diese inkludiert auf einem einzigen Chip ARM Cores, FPGAs, Shared Memory und Peripheriebausteine.

Die Durchführung von MBD auf All-Programmable SoC basierenden Systemen ist keine einfache Herausforderung [3, 4]: Wir haben im Laufe von 6 Jahren beachtlich in diesen Themenbereich investiert, um Tools und kundenspezifische Hardware zu entwickeln und diese stetig weiter zu verbessern [2]. Dafür wurde an unserem Institut ein Team gebildet, das auf model-based realtime Computing spezialisiert ist. Die Hauptaufgaben seien wie folgt zusammengefasst:

- Entwicklung echtzeitfähiger Modelle in MATLAB/Simulink für Signalverarbeitung und Regelung vor allem im Bereich Leistungselektronik und elektrischer Antriebe

- Angebot eines Workflows, der eine nahtlose Entwicklung von Modellen auf soft-Cores und FPGAs unserer Echtzeitplattform, die auf ZYNQ Architekturen basiert, ermöglicht

- Entwicklung einer graphischen Benutzerschnittstelle in NI LabVIEW und deren Verbindung via standardisierter Kommunikationsprotokolle zu unserer Echtzeitplattform

- Entwicklung von Embedded Linux Anwendungen für on-chip Monitoring und massives Data-Processing

Das Model-Based Realtime Computing Team bietet:

- Eine vielfältige Gruppe erfahrener Ingenieure, junger Absolventen und Studenten
- Wertvolles Knowhow, das durch zahlreiche Kooperationsprojekte vertieft wurde

- Zugang zu den neusten Entwicklungstools

Als Beispiel für unsere Entwicklungen auf dem Gebiet des modellbasierten realtime Computings sei ein Hardware-in-the-Loop (HIL) Demonstrator einer Permanentmagnet-Synchronmaschine (PMSM) zusammen mit ihrer Regelung basierend auf Field Oriented Control (FOC) angeführt. In diesem Demonstrator sind zwei ZYNQbasierende Entwicklungsboards über Echtzeitsignale verbunden. Eines der beiden Boards integriert das Modell eine PMSM zusammen mit einem vereinfachten Modell einer Inverters in seinem FPGA, das andere beinhaltet im entsprechenden FPGA den Kontrollalgorithmus, welcher die Geschwindigkeit und das Drehmoment des Motors regelt.

Abbildung 1(a) zeigt die Implementierung eines der Modelle in MATLAB/Simulink und die integrierte Toolchain für den nahtlosen Einbau des generierten Codes in die Echtzeitplattform, in Abb. 1(b) wird der Prototyp gezeigt und in Abb. 1(c) das Blockdiagramm der Schnittstellen.

\section{F\&E Schwerpunkt Programmierbare Logik (FPGA) und} Realtime Computing

Der Schwerpunkt in dieser F\&E-Tätigkeit bezieht sich hauptsächlich auf den Einsatz der Xilinx ZYNQ und Ultrascale ZYNQ System-OnChip Komponenten, welche neben einem FPGA auch mehrere ARM 
Mikroprozessoren beinhalten. Neben der Entwicklung elektronischer Schaltungen im FPGA wird auch Embedded Software basierend auf dem Betriebssystem Linux eingesetzt, um komplette Systemfunktionen zu realisieren. Die derzeitigen Anwendungsfelder für das FPGA Design sind vor allem Implementierungen von sehr rechenintensiven Algorithmen, z.B. [5], für Bildverarbeitung und Signalverarbeitung.

\subsection{Projekte im Bereich der Bildverarbeitung}

Im Projekt MAMMOBEE [6] geht es um die Implementierung eines Algorithmus' aus der Bionik, entwickelt am Institut für Zoologie, Karl-Franzens-Universität Graz von Prof. Manfred Hartbauer. Dieser Algorithmus wurde entwickelt, um stark unterbelichtete Röntgenbilder so zu entrauschen, dass feine Bilddetails erhalten bleiben und in Regionen mit wenigen Bilddetails das Rauschen stärker unterdrückt wird, was mit einer adaptiven Tiefpassfilterung erreicht wird. Namensgebend für diesen Algorithmus ist die nachtaktive Biene Megalopta Genalis aus Mittelamerika, die in der Lage ist, in nahezu absoluter Dunkelheit Photonen im Auge aufzusammeln und so ihre Nachtsicht erreicht. Sinngemäß kann der Mammobee-Algorithmus das Maß an benötigter Röntgenstrahlung in der Mammographie bei gleichbleibender Bildqualität reduzieren. Wegen der hohen Auflösung der Röntgenbilder (ca. 3500 x 2000 Pixel bei 16-bit Graustufen im DICOM Format) und der Komplexität des adaptiven Tiefpassfilters zur Rauschunterdrückung wurde für die Implementierung eine ZYNQ Ultrascale Plattform ausgewählt. Nach einer Vorverarbeitung wird der Bildinhalt von ca. 20 Megapixeln mittels DMA (Direct Memory Access) in mehreren Iterationen im FPGA verarbeitet, wobei die massive Parallelverarbeitung eine beträchtliche Performancesteigerung gegenüber einem Desktop Computer erzielt. Das adaptive Filter prüft in Bereichen mit ansteigendem Radius um ein Zentralpixel die Varianz der Bildhelligkeiten bis zu einem Radius von 15 Pixeln solange, bis diese ermittelte Varianz einen vorgegeben Schwellenwert übersteigt und anschließend wird dieser Pixelbereich gemittelt. Damit bleiben Bilddetails (hohe Varianz) von der Tiefpass- Filterung ausgenommen. Für den Prototypen des Demonstrators wird ein UItrascale SoM (System-On-Module) Trägerboard der Firma Trenz mit Displayport-, Ethernet, USB Schnittstelle zusammen mit einem 4K UHD Monitor verwendet. Fördergeber für das Projekt ist das AWS (Austria Wirtschaftsservice).

Ein weiteres Projekt, BIOKOLLAVOID, dient zur Umsetzung eines bionischen Antikollisionsdetektors für Drohnen (UAVs) in Zusammenarbeit mit dem Institut für Zoologie der Karl-FranzensUniversität Graz. Es wurde von der FFG bereits genehmigt und startete im Oktober 2019. Auf einer Hardware-Plattform mit Xilinx ZYNQ System-On-Chip, 2 Global Shutter Cameras mit 120 frames/sec soll ein Algorithmus, welcher auf der Funktion der Facettenaugen von Wanderheuschrecken basiert, im FPGA realisiert werden. Ein aus einem SIMULINK Modell mittels C-Code Generierung entworfener Flugregler soll weiters auf einer ARM-CPU laufen und über WiFi parametrierbar sein. Dieses Modell wird vom Institut für Luftfahrt und Aviation (LAV) der FH JOANNEUM auf einem Virtual Reality Prüfstand von Fraunhofer entwickelt, um den vom Antikollisionsalgorithmus errechneten Ausweichvektor zu implementieren, der ein automatisches Ausweichen im Falle einer drohenden Kollision mit anderen Objekten ermöglicht, ähnlich dem Verhalten von Wanderheuschrecken in einem Schwarm. In Zusammenarbeit mit der Firma Drone-Rescue sollen auch praktische Feldversuche durchgeführt werden.

\subsection{Projekte im Bereich der Signalverarbeitung}

Das bereits abgeschlossene Projekt DECODE [7] in Zusammenarbeit mit Teleconsult Austria (TCA), gefördert von der FFG, hatte zum
Ziel, einen low-cost GNSS Signal Spoofer mit Hardware-Kosten unter $€ 1.000$ aufzubauen, mit dem GPS-basierte Endgeräte wie Drohnen, Smart Phones, usw. beeinträchtigt werden. Ziel war, ein Bewusstsein für die Gefahren von GNSS Attacken zu entwickeln. Dabei wurden in einer Simulation errechnete GNSS Signale auf einen Träger im L1 Band $(1575,42 \mathrm{MHz})$ aufmoduliert und synchron zu den live GPS Signalen abgestrahlt; in einem Feldversuch konnte tatsächlich eine Drohne von ihrer Position abgelenkt werden.

Im Projekt GIDAS, beauftragt von der Europäischen Weltraumbehörde ESA, wird zurzeit in Zusammenarbeit mit Teleconsult Austria ein Empfänger für GNSS Signale (GPS, Galileo, Glonass, Beidu) auf einer Xilinx Ultrascale ZYNQ Hardware Plattform entwickelt, der eine detaillierte Analyse der empfangenen Satellitensignale gestattet, um mögliche Spoofing und Jamming Angriffe zu detektieren. Der Beitrag des Instituts für Electronic Engineering ist die Entwicklung des digitalen GNSS Empfängers, dessen Herzstück ein Blockkorrelator ist, der für alle Satellitenkanäle die gesamten Korrelationsfunktionen der lokalen Code-Replica mit den empfangenen Funksignalen errechnet, im Falle von GPS sind das bis zu 16 Korrelationsfunktionen mit 4092 Samples pro Millisekunde, was einer Rechenleistung von ca. 256 GOPs (1 Operation = Multiply/Accumulate) entspricht. Das erlaubt das sofortige Erkennen von Fake-Signalen, die den tatsächlichen Satellitensignalen auch zeitlich verschoben überlagert werden. Zwei Realtime ARM R5 Prozessoren und ein Quad A45 Arm Core komplettieren die Funktion der GNSS Interference Detection and Analysis Plattform.

Das Projekt ENTRANCE war eine Kooperation mit der TU Graz im Rahmen eines FFG Headquarter-Programmes. Es wurden unter anderem Vorverzerrungsstrukturen für WLAN 802.11ac untersucht. Dafür wurden neue Signalverarbeitungsmethoden entwickelt und prototypisch umgesetzt. Mit den entwickelten Methoden konnte auch die IEEE Microwave Theory and Techniques Society Student Design Competition „PA Linearization Through DPD" 2017 auf der IEEE MTT-S International Microwave Symposium (IMS) gewonnen werden [8], wobei einer der beteiligten Doktoranden ein Absolvent unseres ehemaligen Studienganges „Industrielle Elektronik" ist.

\section{Elektromagnetische Verträglichkeit}

Auf die elektromagnetische Verträglichkeit sowohl in theoretischer Betrachtung als auch in Hinblick auf die messtechnische Praxis wird bereits seit Gründung des Studienganges Industrielle Elektronik im Jahre 1995 ein überaus großer Fokus gelegt. Der Umstand, dass Studierende in ihrer beruflichen Praxis in der Entwicklung von elektronischer Hardware bzw. der hardwarenahen Software tätig sein werden, bedeutet die Notwendigkeit des fachlichen Wissens über die Entwicklung von Elektronik und auch ein grundlegendes Wissen über die rechtliche Situation zur Zulassung dieser Produkte. Das Bewusstmachen, dass neben der Erfüllung der Funktionalität eines Gerätes ein durchaus nicht vernachlässigbarer Aufwand in die Konformität mit den zu erfüllenden Richtlinien eingebracht werden muss, ist Grundkonsens am Institut.

Nach wie vor ist es leider auch in der industriellen Praxis nur allzu oft der Fall, dass Produktentwicklungen zu stark sequenziell abgearbeitet werden, was bedeutet, dass der Fokus bei der Entwicklung möglicherweise ressourcenbedingt zu lange ausschließlich auf der Funktionalität liegt. Der dauernde Zeitdruck, sich rasch ändernde Marktvorgaben aufgrund geänderter Rahmenbedingungen und das ewige Problem des Mangels an Manpower führen dazu, dass Entwickler oft ohne große Unterstützung an der Lösung ihrer mitunter sehr komplexen funktionellen Aufgaben arbeiten, aber dabei kaum oder im ungünstigsten Fall völlig auf mögliche Anforderungen hinsichtlich der Gerätesicherheit, der elektromagnetischen Kompatibi- 
lität oder andere auf das Produkt zutreffende Richtlinien „vergessen". Die Produkte kommen am Ende der Entwicklungsphase in das Prüflabor und sollen nach Abschluss der notwendigen Prüfungen möglichst unverzüglich verkauft werden können, so der Plan. Aber leider kommt genau an diesem Punkt mitunter oft das böse Erwachen und es werden plötzlich noch Redesigns und Unmengen an zusätzlichen Arbeits- und Prüfstunden erforderlich, ausschließlich um die rechtlichen Anforderungen an das Produkt zu erfüllen. Die Folge? Der geplante Markteintritt wird dramatisch verzögert, was mit ausbleibenden Umsätzen durch den verspäteten Produktstart und mit zusätzlichen enormen Kosten durch den ungeplanten Entwicklungsaufwand verbunden ist. Wie oft konnten wir dieses Szenario in einer mehr oder weniger deutlichen Ausprägung schon beobachten?

25 Jahre Erfahrung in der Entwicklung elektronischer Geräte am Institut Electronic Engineering haben sich insofern auf die Lehre ausgewirkt, dass den Auszubildenden neben dem Umgang mit industrienahen CAD Tools auch die Fertigungstechnologien der zu entwickelnden Hardware über eine enge Zusammenarbeit mit dem Leiterplattenhersteller AT\&S unmittelbar nähergebracht werden. Dabei geht es um Verfahren, Materialien, Aufbauten etc. für verschiedenste Arten von Leiterplatten. Grundlegende Designrules, um optimale Fertigungsprozesse zu nutzen, Maßnahmen zur thermischen Anbindung von Powerelektronik, bis hin zu aktuellen Trends wie das Embedding von passiven und aktiven Bauteilen müssen in der Lehre ihren Platz finden.

Zusätzlich wird neben der Schaltungsentwicklung ein massiver Schwerpunkt auf störfestes und emissionsarmes Elektronikdesign gesetzt. Die Ursachen für Störemissionen werden theoretisch und anhand von praktischen Beispielen in den Lehrveranstaltungen erarbeitet. Ziel ist es, Ursache und Auswirkungen zu begreifen und ein Gefühl für die schlussendlich oft nicht triviale Problemstellung zu bekommen.

Neben der Funktion der Schaltungen müssen den Auszubildenden grundlegende Maßnahmen bekannt sein, um gestrahlte und leitungsgeführte Emissionen zu reduzieren bzw. die Störfestigkeit einer Baugruppe, sowohl bei der Hardware als auch im Softwaredesign, zu erhöhen. Jede aufgebaute Schaltung wird zum Abschluss der Lehrveranstaltung Gerätedesign im institutseigenen HF und EMV Labor in Form von Übungen entsprechend der zutreffenden Produktnorm analysiert und durch Maßnahmen nachträglich optimiert. Dieser Ansatz war neben der Lehre auch dem Kompetenzaufbau am Institut sehr dienlich. Die kontinuierliche Forschung in diesem Bereich hat zu mittlerweile langjährigen Kooperationen mit namhaften Firmen geführt - nicht nur über die Zusammenarbeit mit Studenten, sondern auch direkt eingebunden als Kompetenzpartner im Produktentwicklungsprozess.

Am Institut wird eine voll automatisierte und videoüberwachte $7 \times 4 \times 3$ m Vollabsorber Schirmkammer (Abb. 2) zur Messung von Emissionen bis $18 \mathrm{GHz}$ und gestrahlter Störfestigkeit bis $6 \mathrm{GHz}$ mit Feldstärken von $30 \mathrm{~V} / \mathrm{m}$ betrieben. Daneben gibt es einen Antennenmessplatz, einen Prüfplatz für leitungsgeführte Emissionen und Prüfplätze für alle gängigen leitungsgeführten Störfestigkeitsprüfungen. Zusätzliches Equipment für die Charakterisierung von elektronischen Komponenten, eine Kammer für Temperaturmessungen, Nahfeldscanner, Prüfsonden und eine Vielzahl an speziellen Prüfaufbauten bieten die Basis für Forschung und effiziente Problemlösung im Entwicklungsprozess.

Die Forschungen am Institut im Bereich der EMV sind zu einem Teil Auftragsarbeiten mit relativ zielgerichteten kundenspezifischen Anforderungen und zum anderen Teil öffentlich finanzierte Forschungen sehr verschränkt gestaltet mit den Aktivitäten im Bereich der

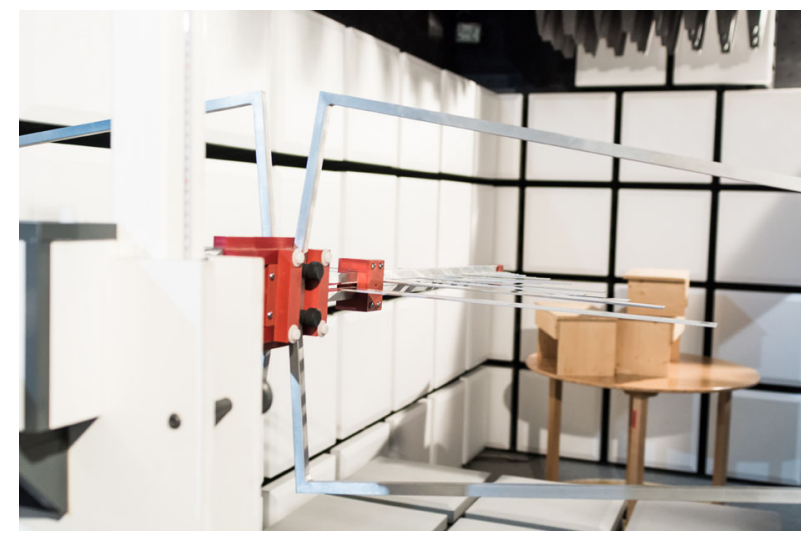

Abb. 2. Vollabsorber Schirmkammer

Forschungen auf dem Gebiet der Leistungselektronik. In Abstimmung mit den Anforderungen der Leistungselektronik wurde das Equipment peu à peu sowohl bei den leitungsgeführten Emissionen und der leitungsgeführten Störfestigkeitsmessungen auf dreiphasige Systeme bis zu $600 \mathrm{~A}$ und $690 \mathrm{~V}$ erweitert.

Aktuelle Forschungen an DC/DC GaN Convertern $[9,10]$ mit Zwischenkreisspannungen von 650VDC bei Schaltgeschwindigkeiten bis $1 \mathrm{MHz}$ befassen sich mit den unterschiedlichen Einflüssen von passiven Komponenten unterschiedlicher Fertigungstechnologien auf die Höhe der abgestrahlten Emissionen in einem Frequenzbereich bis $1 \mathrm{GHz}$. Die Notwendigkeit energieeffizienter, kompakter Designs mit möglichst geringem Gewicht, hohen Schaltgeschwindigkeiten und großen Spannungshüben führt aufgrund von parasitären Eigenschaften der Bauteile bzw. des Aufbaues zwangsweise zu Problemen bei Emissionen. Ohne gezielte Maßnahmen zur Reduktion der parasitären Eigenschaften von Komponenten, Layout und System inklusive einer speziellen Aufbautechnik ist es praktisch nicht möglich, Geräte außerhalb einer geschützten Laborumgebungen zum Einsatz zu bringen. Geringe kapazitive Ankopplungen an Kühlkörper, auf die maximal zulässigen Verluste abgestimmte, vom Wert so klein wie möglich und so groß wie nötige Gatewiderstände, mit möglichst hoher Schaltfrequenz betriebene, minimale induktivitätsarme Stromschleifen und spezielle und äußerst kompakte Aufbautechniken mit neuen speziellen Werkstoffen sind die notwendigen Ingredienzien, um die Leistungselektronik effizient aber auch die elektromagnetischen Störungen beherrschbar zu machen.

Neben der Leistungselektronik stellt auch die drahtlose, energieautarke Sensorik ein zweites aktuelles Forschungsthema dar. Hier wird basierend auf dem Prinzip der kapazitiven Detektion von Vereisungen an Oberflächen, wie beispielsweise Rotorblättern an Windrädern, Flugzeugtragflächen, Eisenbahnweichen, etc., untersucht [11-13], wie die Funkübertragung, das Energieharvesting und die eigentliche Sensorik so kombiniert werden können, dass damit möglichst keine "Blind Spots"(dies sind Flächen des Sensors, die für die Funktion des Sensors notwendig sind, aber sensorisch nicht aktiv sind) entstehen $[14,15]$ und die Systeme dennoch störfest und mit ausreichender Performance funktionieren $[16,17]$.

\section{Wissenstransfer in die Lehre}

Leistungselektronik, Realtime Computing, FPGA, EMV - diese Fachgebiete nehmen neben anderen wie Mixed-Signal Systeme [18], Automotive Control, Automatisierung etc. auch jeweils zentrale Schwerpunkte bei der Ausbildung am Institut Electronic Engineering ein.

Im Wesentlichen können F\&E-Aktivitäten auf drei Arten Einfluss auf die Studiengänge nehmen [19]: 
- Ergebnisse oder Erkenntnisse aus F\&E-Projekten werden direkt in die Gestaltung von Lehrveranstaltungen übernommen: Beispiele sind praxisorientierte Fallstudien, die oft nur Teilaspekte eines F\&E-Projektes beleuchten und doch die Anwendung von Lehrinhalten veranschaulichen. Ein weiterer Aspekt betrifft Lehrmittel: So kann erworbenes Know-How von aktuellen HardwareKomponenten und Softwareprodukten in der Lehre - vor allem auch in Laborübungen - genutzt werden. Beispiele dafür sind Microcontrollersysteme mit den dazugehörigen Entwicklungsumgebungen, CAD-Systeme oder die Plattformen zur Entwicklung von FPGAs.

- Durch F\&E-Projekte werden neue Entwicklungen aufgezeigt, die dann in einer längerfristigen Planung von Lehrinhalten und Curricula berücksichtigt werden können. Viele F\&E-Projekte ermöglichen die Arbeit an zukunftsweisenden Technologien, die für die Entwicklung inhaltlicher Schwerpunkte eines Studiengangs Relevanz gewinnen können. Dazu zählen die Verwendung automatischer Code-Generierung bei der Programmierung von eingebetteten Systemen oder der Einsatz von Halbleitern aus Siliziumcarbid und Galliumnitrid in der Leistungselektronik $[20,21]$.

- Laborinfrastruktur, die für einen reinen Einsatz in der Lehre zu kostspielig wäre, kann durch Nutzung in F\&E-Projekten finanzierbar werden. Am Institut Electronic Engineering konnte durch solche Doppelnutzung das HF-Labor mit Schirmkammer sowie die erforderliche Mess-Ausstattung und das Labor für Leistungselektronik eingerichtet werden.

Auf diese Weise ergänzen sich in den jeweiligen Kompetenzbereichen des Institutes die Forschungsaktivitäten, Kundenprojekte, Lehre und der Wissenstransfer in die Lehre.

\section{Ausblick}

Zusammenfassend lässt sich sagen, dass das Institut Electronic Engineering seine strategische Ausrichtung der nächsten Jahre auf folgende Themenschwerpunkte abzielt: Forcierung des systemischen Ansatzes in Bezug auf die Forschungsbereiche Leistungselektronik, elektromagnetische Verträglichkeit, PCB- und FPGA-Design, Realtime Computing, Automotive Control und Automatisierung. Verstärkt werden sollen des weiteren Forschungsaktivitäten im Bereich Mechatronik. Diese Vorgehensweise passiert in ständiger Abstimmung mit der Transformation des gewonnenen Wissens in die Lehre und daraus resultierend in die Weiterentwicklung unserer Curricula. Vor Kurzem wurde das Masterstudium System Test Engineering zur Akkreditierung eingereicht. Langfristig soll dadurch der positive Ruf unseres Ausbildungsprogrammes sowie des Forschungszentrums nachhaltig gefestigt und weiter ausgebaut werden, um auch immer mehr junge Menschen von diesem innovativen Forschungsfeld überzeugen zu können.

\section{Danksagung}

Open access funding provided by FH Joanneum - University of Applied Sciences.

Hinweis des Verlags Der Verlag bleibt in Hinblick auf geografische Zuordnungen und Gebietsbezeichnungen in veröffentlichten Karten und Institutsadressen neutral.

Open Access Dieser Artikel wird unter der Creative Commons Namensnennung 4.0 International Lizenz veröffentlicht, welche die Nutzung, Vervielfältigung, Bearbeitung, Verbreitung und Wiedergabe in jeglichem Medium und Format erlaubt, sofern Sie den/die ursprünglichen Autor(en) und die Quelle ordnungsgemäßnennen, einen Link zur Creative Commons Lizenz beifügen und angeben, ob Änderungen vorgenommen wurden. Die in diesem Artikel enthaltenen Bilder und sonstiges Drittmaterial unterliegen ebenfalls der genannten Creative Commons Lizenz, sofern sich aus der Abbildungslegende nichts anderes ergibt. Sofern das betreffende Material nicht unter der genannten Creative Commons Lizenz steht und die betreffende Handlung nicht nach gesetzlichen Vorschriften erlaubt ist, ist für die oben aufgeführten Weiterverwendungen des Materials die Einwilligung des jeweiligen Rechteinhabers einzuholen. Weitere Details zur Lizenz entnehmen Sie bitte der Lizenzinformation auf http://creativecommons.org/licenses/by/4.0/deed.de.

\section{Literatur}

1. Institut für Electronic Engineering, FH JOANNEUM: (2019): URL zugegriffen: Nov. 2019, https://www.fh-joanneum.at/institut/electronic-engineering/.

2. JOANNEUM Power Electronics Center (JPEC): URL zugegriffen: Nov. 2019, https:// www.fh-joanneum.at/projekt/joanneum-power-electronics-center/.

3. Streit, F.-J., Letras, M., Wildermann, S., Hackenberg, B., Falk, J., Becher, A., Teich, J. (2018): Model-based design automation of hardware/software co-designs for Xilinx Zynq PSoCs. In International conference on reconfigurable computing and FPGAs, Mexico, Cancun.

4. Xiong, Z., Li, S., Chen, J. (2005): Hardware/software co-design using hierarchical platform-based design method. In Design automation conference.

5. Midl, M., Netzberger, Ch. (2009): Hardware acceleration of RNA folding. In Austrochip 2009 (S. 109-115). Graz: TU Graz. ISBN 978-3-9501635-1-3.

6. Mammobee. URL zugegriffen: Nov. 2019, https://www.fh-joanneum.at projekt/mammobee/.

7. DECODE. URL zugegriffen: Nov. 2019, https://www.fh-joanneum.at/projekt/decodespoofing-attacken/.

8. Enzinger, H., Freiberger, K., Vogel, C. (2018): Competitive linearity for envelope tracking: dual-band crest factor reduction and 2D-vector-switched digital predistortion. IEEE Microw. Mag., 19(1), 69-77.

9. Stocksreiter, W., List, H., Berger, H., Weis, G., Krenn, M., Engel, G. (2016): Electromagnetic emissions in high density and fast GaN switched half bridges with resonance filter structures. In International exhibition and conference for power electronics, intelligent motion, renewable energy and energy management, PCIM Europe 2016 (S. 2151-2158). https://ieeexplore.ieee.org/document/7499617. ISBN 978-38007-4186-1.

10. Fernandez-Serantes, L. A., Berger, H., Stocksreiter, W., Weis, G. (2016): Ultra-high frequent switching with GaN-HEMTs using the coss-capacitances as non-dissipative snubbers. In International exhibition and conference for power electronics, intelligent motion, renewable energy and energy management, PCIM Europe 2016 (S. 296-303). https://ieeexplore.iee.org/document/7499371. ISBN 978-3-8007-4186-1.

11. Richards, T. (2012): ON-WING - on wing ice detection and monitoring system. Project final report ACP8-GA-2009-233838 - Framework 7. European Commission.

12. Schlegl, T., Moser, M., Zangl, H. (2015): Wireless and flexible ice detection on aircraft. SAE technical paper 2015-01-2112. https://doi.org/10.4271/2015-01-2112.

13. Moser, M., Schlegl, T., Zangl, H. (2014): Retrofittable, autonomous and wireless icing and temperature monitoring on rotor blades for efficient anti- and de-icing. In Winterwind 2014: International wind energy conference.

14. Stocksreiter, W., Zangl, H. (2018): Kombinierte Sensor- und Kommunikationseinrichtung. Patent application A 264/2018.

15. Lenzhofer, M. Stocksreiter, W., Szirch, St. (2017): A new approach for in-line hea chuck temperature measurement based on SAW technology. In MikroSystemTechnik 2017 congress, ISBN 978-3-8007-4491-6.

16. Stocksreiter, W., Zangl, H., Moser, M. (2017): Environmental impacts on antennas for wireless sensors on outer aircraft surfaces. In 2017 IEEE international workshop on metrology for aerospace, MetroAeroSpace, (pp. 21-23). https://doi.org/10.1109/ MetroAeroSpace.2017.7999625.

17. Stocksreiter, W., Zangl, H., Kaur, M., Glaser, D., Moser, M. (2018): Compact wireless ice detection system with electrode used as antenna. In IEEE world congress of the international measurement federation. IMEKO 2018. J. Phys. Conf. Ser. (1065). Technical diagnostics

18. Vogel, C., Enzinger, H., Freiberger, K. (2019): Digitally enhanced mixed signal systems - the big picture. In C. Jabbour, P. Desgreys, D. Dallet (Hrsg.), Digitally enhanced mixed signal systems, London: The Institution of Engineering and Technology (S. 1-25). Ch. 1.

19. Salloker, M. G., Okorn, R. (2011): Der Einfluss von Forschungs- und Entwicklungstätigkeit auf FH-Studiengänge. In K. P. Pfeiffer, H. Burgsteiner (Hrsg.), Festschrift für Helfrid Maresch (S. 133-149). Leycam: FH \& Health-Care Engineering.

20. Obermayr, W. (2017): Bye-bye Silizium: Wide-Bandgap-Halbleiter übernehmen die Leistungselektronik. Elektron. Ind. 5, 58-62. Heidelberg: Hüthig-Verlag. ISSN 01745522. http://www.all-electronics. de/wide-bandgap-halbleiter/.

21. Obermayr, W., Gebeshuber, I. C. (2010): Nanoscale science and engineering. In Guston, D.H. (Hrsg.), Encyclopedia of nanoscience and society. Thousand Oaks: Sage Publications. 
Autoren

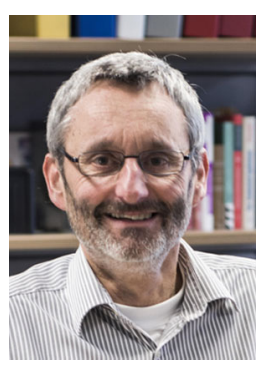

\section{Hubert Berger}

FH-Prof. Dipl.-Ing. Dr., schloss sein Diplomstudium in Industrieller Elektronik und Regelungstechnik an der Technischen Universität Wien im Jahr 1986 ab. Danach war er als Wissenschaftlicher Mitarbeiter am Institut für elektrische Maschinen und Antriebe/Prof. Kleinrath tätig und promovierte im Jahr 1990 im Bereich drehzahlgeregelter Drehstromantriebe. Nach seiner Zeit als Projektleiter be VA-Tech-ELIN trat er 1996 als Lehrender für Leistungselektronik und elektrische Antriebe in die FH JOANNEUM ein. Hubert Berger leitete von 2005 bis 2014 das Institut für Electronic Engineering und den zugehörigen Bachelor- sowie Masterstudiengang. Sein Forschungsschwerpunkt liegt im Bereich hocheffizienter Leistungselektronik.

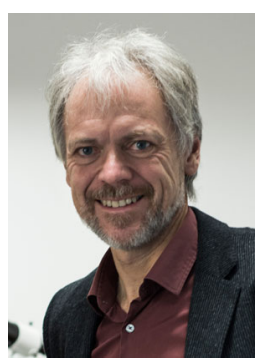

\section{Christian Netzberger}

FH-Prof. Dipl.-Ing. Dr., studierte Elektrotechnik an der Technischen Universität in Graz mit der Vertiefungsrichtung Elektronik und Nachrichtentechnik und beendete das Studium 1988. Die Diplomarbeit beinhaltete den Entwurf und Aufbau eines Spread-Spectrum Satellitenmodems. Von 1987-1998 arbeitete er zunächst am Institut für Nachrichtentechnik und Wellenausbreitung der TU Graz und später am Institut für Angewandte Systemtechnik des Joanneum Research an verschiedenen wissenschaftlichen ESA-Projekten. 1999 beendete er seine Dissertation mit dem Titel „Design and Implementation of a Portable Satellite Communications System". Seit 1998 ist er Dozent an der Fachhochschule FH JOANNEUM in Kapfenberg am Studiengang Electronics and Computer Engineering. Die Vorlesungen beinhalten Digitale Schaltungstechnik, VHDL-Design, Nachrichtentechnik und Kommunikationstechnologie. Neben der Lehre ist er in der Abwicklung industrieller Projekte tätig.

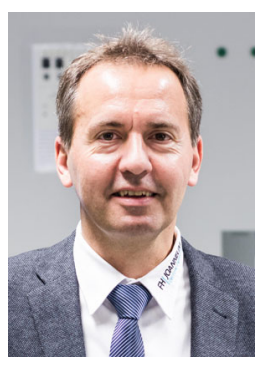

\section{Wolfgang Stocksreiter}

Dipl.-Ing. (FH), ist Dozent am Institut Electronic Engineering (IEE) an der FH JOANNEUM. Er studierte Elektronik/Elektrotechnik an der Fachhochschule JOANNEUM und der TU Graz. Von 1990 bis 2000 arbeitet er als Entwicklungsingenieur bei der ATB Austria Antriebstechnik, seit 2000 ist er an der Fachhochschule JOANNEUM beschäftigt. Er ist im Bereich Hochfrequenz und Elektromagnetische Verträglichkeit tätig und leitet das am Institut angesiedelte EMV Labor. Neben der Abwicklung zahlreicher Industrieprojekte schwerpunktmäßig im Themenfeld Consulting für Sicherheit und EMV und der Durchführung von precompliance Messungen im Produktentwicklungsprozess, war er auch in verschiedenen Forschungsprojekten im Themenfeld drahtlose Kommunikation und Antennendesign involviert. Er ist gerichtlich beeideter Sachverständiger für elektrische Antriebe, Niederspannungsanlagen und Elektromagnetische Verträglichkeit sowie zertifizierter Projektmanager IPMA Level B.

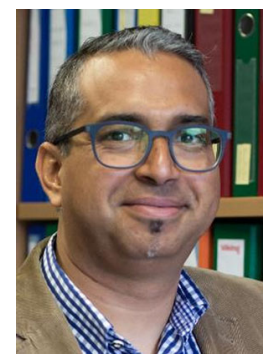

Raúl Estrada-Vázquez

BSC, MMSc, studierte Elektrotechnik (BSc) an der Universität "Instituto Tecnológico de Veracruz", in Mexiko. Von 1998 bis 1999 war er als technischer Assistent für nukleare Dampfversorgungssysteme bei "Laguna Verde Nuclear Power Station" in Mexiko tätig. Als wissenschaftlicher Assistent an der Universität "Tecnológico de Monterrey", Mexiko, schloss er 2002 sein Master-Studium (MSc) für Automationstechnik ab und arbeitete bis 2006 als Universitätsund Forschungsassistent im Bereich der industriellen Prozessautomatisierung und Steuerungstechnik. Von 2006 bis 2008 beschäftigte er sich bei ADEX S.L., Adaptive Predictive Expert Control, Madrid, als Entwickler und Projektleiter mit der Steuerung und Optimierung industrieller Prozesse in Simulation und Anwendung. Seit Juni 2009 ist er Mitarbeiter an der FH JOANNEUM, wo er 2012 das Master-Studium für "Advanced Electronics Engineering" abschloss. Derzeit forscht und lehrt er als Dozent am Institut Electronic Engineering auf dem Gebiet des Modellbasierten Designs für EchtzeitComputing. Sein besonderes Interesse gilt neuen Konzepten von Leistungselektronik-Wandlern für Elektromobilität und Energieeffizienz.

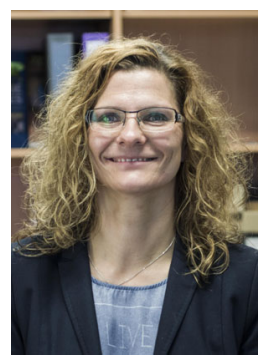

Nina Blasonig

Mag., absolvierte im Jahr 2000 ihr Studium "Europa - Sprachen, Wirtschaft, Recht" an der Karl-Franzens-Universität Graz. AnschlieBend war sie fast 10 Jahre lang in der Pankl Gruppe im Verkauf und als Assistentin der Geschäftsführung beschäftigt. Die Firma produziert Motoren- und Antriebsteile für Rennund Luxusautos. Schon während dieser Zeit arbeitete sie in Förderprojekten mit. Seit 2009 ist Frau Blasonig an der FH JOANNEUM beschäftigt und für Projektmanagement und -controlling, sowie für internationale Beziehungen am Institut „Electronic Engineering" zuständig. Sie unterrichtet „Intercultural Communication" im Master.

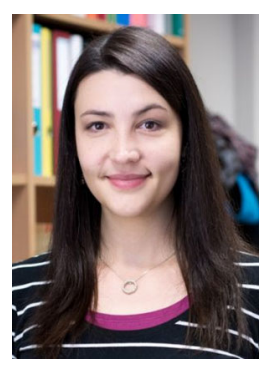

\section{Aida Preda}

MSc, studierte von 2007 bis 2011 Nachrichtentechnik und Elektronik an der Technischen Universität „Politehnica Timişoara" in Rumänien. Im Jahr 2014 schloss sie ihr Masterstudium an der FH JOANNEUM mit dem Schwerpunkt Automotive Elektronics erfolgreich ab. Seit Abschluss ihres Studiums ist sie als Wissenschaftlerin an der FH JOANNEUM in Kapfenberg im Bereich der digitalen Hardwareentwicklung, Simulation und Steuerungstechnik tätig. Derzeit ist sie an internationalen Projekten, wie OBELICS und HiPERFORM, beteiligt und konzentriert ihre Forschung auf die Entwicklung effizienter Hardware-Software Co-Design-Methoden. 


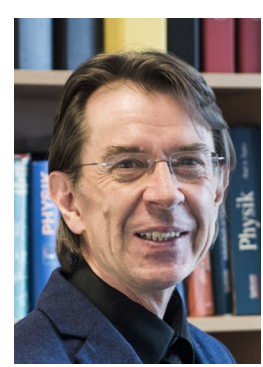

\section{Werner Obermayr}

FH-Prof. Dipl.-Ing. Dr., schloss sein Studium der Technischen Physik an der Technischen Universität in Graz im Jahr $1988 \mathrm{ab}$. Nach der beruflichen Station bei der Firma „CAS - Computer, Applikationen, Software"absolvierte er ein Doktoratsstudium auf dem Gebiet der Theoretischen Festkörperphysik. 1991 beendete er seine Dissertation mit dem Titel "Berechnung impulsabhängige Elektron-Positron Annihilationsraten in Kristallen". Anschließend war er als Universitäts- und Forschungsassistent am Institut für Kernphysik der TU Graz tätig. Seit 1996 ist er an der FH JOANNEUM am Institut für Electronic Engineering tätig. Seine Lehr- und Forschungsinteressen betreffen mathematische und physikalische Methoden, derzeit v.a. elektromagnetische Systeme und Halbleiter in der Leistungselektronik.

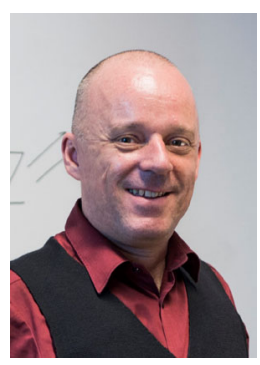

\section{Michael Salloker}

FH-Prof. Dipl.-Ing., absolvierte 1994 das Studium der Elektrotechnik mit dem Schwerpunkt Regelungstechnik und Prozessautomatisierung an der Technischen Universität Graz. Danach arbeitete er als wissenschaftlicher Mitarbeiter bei JOANNEUM RESEARCH. 1998 begann er mit der Tätigkeit an der FH JOANNEUM am damaligen Diplomstudiengang Industrielle Elektronik in der Lehre und in F\&EProjekten, wobei er zum Aufbau des Transferzentrums beitrug. Die von ihm abgehaltenen Lehrveranstaltungen umfassen die Grundlagen der Elektrotechnik (die er zeitweise an insgesamt fünf verschiedenen Instituten der FH JOANNEUM lehrte) sowie die Regelungsund Automatisierungstechnik. Darüber hinaus unterstützt er die Studiengangsleitung bei der Weiterentwicklung der Studienangebote.

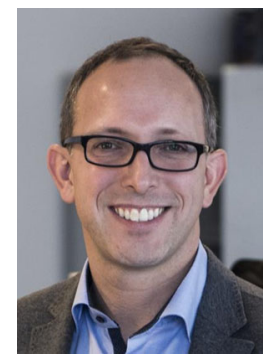

\section{Christian Vogel}

FH-Prof. Priv.-Doz. Dipl.-Ing. Dr., Senior Member IEEE, ist Leiter des Instituts für Electronic Engineering der FH JOANNEUM und FHProfessor für elektronische Systeme. Nach beruflichen Stationen am Institut für Angewandte Informationsverarbeitung und Kommunikationstechnologie, am Kompetenzzentrum Evolaris und bei Infonova BearingPoint absolvierte er sein Doktoratsstudium an der TU Graz. Nach Forschungsaufenthalten an der Universität Linköping sowie an der ETH Zürich wurde Dr. Vogel Key Researcher am Forschungszentrum Telekommunikation Wien, wo er die Gruppe Mixed-Signal Processing leitete und für den Geschäftsbereich Verkehrstelematik verantwortlich war. Seit 2014 ist Christian Vogel an der FH JOANNEUM. Sein Forschungsinteresse liegt unter anderem in der effizienten Umsetzung von komplexen Algorithmen auf eingebettete Rechnersysteme und in deren Vernetzung und Interaktion mit der Umwelt. Er habilitierte sich im Fach „Analoge und digitale Signalverarbeitung ", veröffentlichte über 80 Fachartikel in Zeitschriften, Bücher und in Konferenztagungsbänden und ist Erfinder mehrerer internationaler Patente. 2014 wurde er mit dem IEEE Circuits and Systems Darlington Best Paper Award ausgezeichnet. 